\title{
Fixed point theorems in generalized intuitionistic fuzzy metric spaces using contractive condition of integral type
}

\author{
M. Jeyaraman ${ }^{1 \star}$ and D. Poovaragavan ${ }^{2}$
}

\begin{abstract}
In this paper, we analyze the existence of fixed points for mappings defined on a complete generalized intuitionistic fuzzy metric spaces satisfying a contractive condition of integral type. Our main result generalize the fuzzy Banach contraction theorem.

Keywords

Complete fuzzy metric spaces, Fuzzy Banach contraction, Cauchy sequence, Intuitionistic fuzzy metric spaces.

AMS Subject Classification

47H10, 54H25, 54E50.

${ }^{1} P G$ and Research Department of Mathematics, Raja Dorai Singam Government Arts College, Sivagangai-630561, India.

${ }^{2}$ Department of Mathematics, Government Arts College for Women, Sivagangai-630561, India.

*Corresponding author: ${ }^{1}$ jeya.math@gmail.com; ${ }^{2}$ poovaragavan87@gmail.com

Article History: Received 24 March 2019; Accepted 09 May 2019

(C)2019 MJM.
\end{abstract}

\section{Contents}

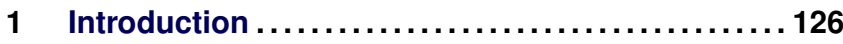

2 Preliminaries ............................... 126

3 Main Results ........................... 127

References .............................. 128

\section{Introduction}

Fuzzy set was defined by Zadeh [11] in 1965 which is a mathematical frame to vagueness or uncertainty in daily life. Kramosil and Michalek [7] introduced fuzzy metric spaces and this concept was modified by George and Veeramani in 1994[4]. Park [8] introduced the notion of intuitionistic fuzzy metric space which is based both on the idea of intuitionistic fuzzy set due to Atanassov [2] and the concept of a fuzzy metric space given George and Veeramani [4]. In 1997 Singh and Chauhan [9] introduced the concept of generalized fuzzy metric spaces known S- fuzzy metric space. In 2000, Bijendra Singh and Chauhan [10] introduced the concept of compatibility in fuzzy metric space. In 2008, Al-Thagafi and Shahzad [1] introduced the notion of occasionally weakly compatible mappings which is more general than the concept of weakly compatible maps. In 2002, an analogue of a Banach contraction principle for integral type inequality Branciari [3] obtained a fixed point theorem for a single mapping.
In this paper, we existence of fixed points for mappings define on a complete generalized intuitionistic fuzzy metric spaces using a contractive condition of integral type. We extend generalized and improved the corresponding results given by many authors earlier given in intuitionistic fuzzy metric spaces.

\section{Preliminaries}

Definition 2.1. The 5- tuple $(X, \mathscr{M}, \mathscr{N}, *, \diamond)$ is said to be generalized intuitionistic fuzzy metric space if $X$ is an arbitrary set. $*$ is a continuous $t$ - norm, $\diamond$ is a continuous t-conorm and $\mathscr{M}, \mathscr{N}$ are fuzzy sets on $X^{3} \times(0, \infty)$ satisfying the following conditions: for every $x, y, z, w \in X$ and $t, s>0$,

$$
\begin{aligned}
& \text { 1. } \mathscr{M}(x, y, z, t)+\mathscr{N}(x, y, z, t) \leq 1, \\
& \text { 2. } \mathscr{M}(x, y, z, t)>0, \\
& \text { 3. } \mathscr{M}(x, y, z, t)=1 \text { iff } x=y=z, \\
& \text { 4. } \mathscr{M}(x, y, z, t)=\mathscr{M}(p\{x, y, z\}, t), \\
& \text { 5. } \mathscr{M}(x, y, z, t+s) \geq \mathscr{M}(x, y, a, t) * \mathscr{M}(a, z, z, s), \\
& \text { 6. } \mathscr{M}(x, y, z, .):(0, \infty) \rightarrow[0,1] \text { is continuous, } \\
& \text { 7. } \mathscr{N}(x, y, z, t)<0,
\end{aligned}
$$



8. $\mathscr{N}(x, y, z, t)=0$ iff $x=y=z$,
9. $\mathscr{N}(x, y, z, t)=\mathscr{N}(p\{x, y, z\}, t)$,
10. $\mathscr{N}(x, y, z, t+s) \leq \mathscr{N}(x, y, a, t) \diamond \mathscr{N}(a, z, z, s)$,
11. $\mathscr{N}(x, y, z,):.(0, \infty) \rightarrow[0,1]$ is continuous.

Example 2.2. Let $X$ is a nonempty set and $D$ is the D-metric on $X$. Denote $a * b=\min \{a, b\}$ and $a \diamond b=\max \{a, b\}$ for all $a, b \in[0,1]$. For each $t \in(0, \infty)$, define

$\mathscr{M}(x, y, z, t)=\frac{t}{t+D(x, y, z)}$ and $\mathscr{N}(x, y, z, t)=\frac{D(x, y, z)}{t+D(x, y, z)}$

for all $x, y, z \in X$. It is easy to see that $(X, \mathscr{M}, \mathscr{N}, *, \diamond)$ is a generalized intuitionistic fuzzy metric space.

Definition 2.3. $\operatorname{Let}(X, \mathscr{M}, \mathscr{N}, *, \diamond)$ be a generalized intuitionistic fuzzy metric space, then

1. A sequence $\left\{x_{n}\right\}$ in $X$ is said to be convergent to $x$ if $\lim _{n \rightarrow \infty} \mathscr{M}\left(x, x, x_{n}, t\right)=1$ and $\lim _{n \rightarrow \infty} \mathscr{N}\left(x, x, x_{n}, t\right)=0$.

2. A sequence $\left\{x_{n}\right\}$ in $X$ is said to be a Cauchy sequence if $\lim _{n, m, p \rightarrow \infty} \mathscr{M}\left(x_{n}, x_{m}, x_{p}, t\right)=1$ and $\lim _{n, m, p \rightarrow \infty} \mathscr{N}\left(x_{n}, x_{m}, x_{p}, t\right)=0$, that is, for any $\varepsilon>0$ and for each $t>0$, there exists $n_{0} \in N$ such that $\mathscr{M}\left(x_{n}, x_{m}, x_{p}, t\right)>1-\varepsilon$ and $\mathscr{N}\left(x_{n}, x_{m}, x_{p}, t\right)<\varepsilon$ for $n, m, p \geq n_{0}$.

3. A generalized intuitionistic fuzzy metric space $(X, \mathscr{M}, \mathscr{N}, *, \diamond)$ is said to be complete if every Cauchy sequence in $X$ is convergent.

Definition 2.4. Let $(X, \mathscr{M}, \mathscr{N}, *, \diamond)$ be a generalized intuitionistic fuzzy metric space. A mapping $T: X \rightarrow X$ is called an intuitionistic fuzzy contractive if there exists

$k \in(0,1)$ such that $\frac{1}{\mathscr{M}(T x, T y, T z, t)}-1 \leq k\left(\frac{1}{\mathscr{M}(x, y, z, t)}-1\right)$ and $\mathscr{N}(T x, T y, T z, t) \leq k \mathscr{N}(x, y, z, t)$ for all $x, y, z \in X$ and $t>0$.

Theorem 2.5. (Fuzzy Banach contraction theorem) Let $(X, M, *)$ be a complete fuzzy metric space in which fuzzy contractive sequences are Cauchy. Let $T: X \rightarrow X$ be a fuzzy contractive mapping being $k$ the contractive constant. Then $T$ has a unique fixed point.

A. Branciari generalized the Banach fixed point theorems as following

Branciari -Integral contractive type condition [3]: For a given $\varepsilon>0$, there exists a real number $\mathrm{c} \in(0,1)$ and a locally Lebesgue-integrable function $\mathrm{g}:[0, \infty) \rightarrow[0, \infty)$ such that $\int_{0}^{d(f x, f y)} g(t) d t \leq c \int_{0}^{d(x, y)} g(t) d t$ and $\int_{0}^{\varepsilon} g(t) d t$, for all $\mathrm{x}, \mathrm{y}$ in $X$ and for each $\varepsilon>0$.

Also, Branciari-Integral contractive type condition is a generalization of Banach contraction map if $g(t)=1$ for all $\mathrm{t} \geq 0$.

\section{Main Results}

Theorem 3.1. Let $(X, \mathscr{M}, \mathscr{N}, *, \diamond)$ be a complete generalized intuitionistic fuzzy metric space, $k \in(0,1)$ and let $T: X \rightarrow$ $X$ be a mapping such that for each $x, y, z \in X$,

$$
\int_{0}^{\left(\frac{1}{\mathscr{M}(T x, T y, T z, t)}-1\right)} \phi(s) d s \leq k \int_{0}^{\left(\frac{1}{\mathscr{M}(x, y, z, t)}-1\right)} \phi(s) d s
$$

and

$$
\int_{0}^{\mathscr{N}(T x, T y, T z, t)} \phi(s) d s \leq k \int_{0}^{\mathscr{N}(x, y, z, t)} \phi(s) d s
$$

where $\varphi:[0, \infty) \rightarrow[0, \infty)$ is a Lebesgue -integrable mapping which is summable on each compact subset of $[0, \infty)$, non negative and such that for each $>0$,

$$
\int_{0}^{1-\varepsilon} \phi(s) d s>0
$$

then $T$ has a unique fixed point $z \in X$ such that $z \in X$, $\lim _{n \rightarrow \infty} T^{n} x=z$.

Proof. Let $x \in X$ be an arbitrary point define a sequence $\mathrm{x}_{n}=\mathrm{T}^{n} \mathrm{x}$. For each integer $n \geq 1$, using (3.1)

$$
\int_{0}^{\left(\frac{1}{\mathscr{M}\left(x_{n}, x_{n+1}, x_{n+1}, t\right)}-1\right)} \phi(s) d s \leq k \int_{0}^{\left(\frac{1}{\mathscr{M}\left(x_{n-1}, x_{n}, x_{n}, t\right)}-1\right)} \phi(s) d s
$$

and

$$
\int_{0}^{\mathscr{N}\left(x_{n}, x_{n+1}, x_{n+1}, t\right)} \phi(s) d s \leq k \int_{0}^{\mathscr{N}\left(x_{n-1}, x_{n}, x_{n}, t\right)} \phi(s) d s
$$

repeating this process $n$ times we get

$$
\int_{0}^{\left(\frac{1}{\mathscr{M}\left(x_{n}, x_{n+1}, x_{n+1}, t\right)}-1\right)} \phi(s) d s \leq k^{n} \int_{0}^{\left(\frac{1}{\mathscr{M}\left(x_{0}, x_{1}, x_{1}, t\right)}-1\right)} \phi(s) d s
$$

and

$$
\int_{0}^{\mathscr{N}\left(x_{n}, x_{n+1}, x_{n+1}, t\right)} \phi(s) d s \leq k^{n} \int_{0}^{\mathscr{N}\left(x_{0}, x_{1}, x_{1}, t\right)} \phi(s) d s
$$

Taking limit $n \rightarrow \infty$ we obtained

$$
\begin{aligned}
& \lim _{n} \int_{0}^{\left(\frac{1}{\mathscr{M}\left(x_{n}, x_{n+1}, x_{n+1}, t\right)}-1\right)} \phi(s) d s=0 \quad \text { and } \\
& \lim _{n} \int_{0}^{\mathscr{N}\left(x_{n}, x_{n+1}, x_{n+1}, t\right)} \phi(s) d s=0 .
\end{aligned}
$$

Which from (3.2) implies

$$
\begin{aligned}
& \lim _{n}\left(\frac{1}{\mathscr{M}\left(x_{n}, x_{n+1}, x_{n+1}, t\right)}-1\right)=0 \quad \text { and } \\
& \lim _{n} \mathscr{N}\left(x_{n}, x_{n+1}, x_{n+1}, t\right)=0
\end{aligned}
$$


which implies

$$
\begin{aligned}
& \lim _{n}\left(\mathscr{M}\left(x_{n}, x_{n+1}, x_{n+1}, t\right)\right)=1 \quad \text { and } \\
& \lim _{n}\left(\mathscr{N}\left(x_{n}, x_{n+1}, x_{n+1}, t\right)\right)=0 .
\end{aligned}
$$

Now we have to show that $\left(x_{n}\right)$ is a Cauchy sequence. Suppose that $\left(x_{n}\right)$ is not a Cauchy sequence. Then there exists $\varepsilon>0$ and sub-sequence $\left(m_{p}\right)$ and $\left(n_{p}\right)$ such that $m_{p} \leq n_{p} \leq m_{p+1}$ with

$$
\begin{gathered}
\mathscr{M}\left(x_{m_{p}}, x_{n_{p}}, x_{n_{p}}, t\right) \leq 1-\varepsilon, \mathscr{M}\left(x_{m_{p}}, x_{n_{p}-1}, x_{n_{p}-1}, t\right)>\varepsilon \text { and } \\
\mathscr{N}\left(x_{m_{p}}, x_{n_{p}}, x_{n_{p}}, t\right)>\varepsilon, \mathscr{N}\left(x_{m_{p}}, x_{n_{p}-1}, x_{n_{p}-1}, t\right) \leq \varepsilon
\end{gathered}
$$

by using (3.3) we get

$$
\begin{aligned}
& \int_{0}^{\left(\frac{1}{\mathscr{M}\left(x_{m_{p},}, x_{m_{p}-1}, x_{m_{p}-1}, t\right)}-1\right)} \phi(s) d s \\
& =\int_{0}^{\left(\frac{1}{\mathscr{M}\left(x_{n_{p}}, x_{m_{p}-1}, x_{m_{p}-1}, t\right)}-1\right)} \phi(s) d s=0 \text { and } \\
& \int_{0}^{\mathscr{N}\left(x_{m_{p}}, x_{m_{p}-1}, x_{m_{p}-1}, t\right)} \phi(s) d s \\
& =\int_{0}^{\mathscr{N}\left(x_{n}, x_{m_{p-1}}, x_{m_{p}-1}, t\right)} \phi(s) d s=0
\end{aligned}
$$

from triangular inequality and (3.4)

$$
\begin{aligned}
& \mathscr{M}\left(x_{m_{p}-1}, x_{n_{p}-1}, x_{n_{p}-1}, t\right) \\
& \geqslant \mathscr{M}\left(x_{m_{p}-1}, x_{n_{p}-1}, x_{m_{p}}, \frac{t}{2}\right) * \mathscr{M}\left(x_{m_{p}}, x_{n_{p}-1}, x_{n_{p}-1}, \frac{t}{2}\right) \\
& \geqslant \mathscr{M}\left(x_{m_{p}-1}, x_{n_{p}-1}, x_{m_{p}}, \frac{t}{2}\right) *(1-\varepsilon) \\
& \rightarrow(1-\varepsilon) \quad \text { as } p \rightarrow \infty \quad \text { and } \\
& \mathscr{N}\left(x_{m_{p}-1}, x_{n_{p}-1}, x_{n_{p}-1}, t\right) \\
& \geqslant \mathscr{N}\left(x_{m_{p}-1}, x_{n_{p}-1}, x_{m_{p}}, \frac{t}{2}\right) \diamond \mathscr{N}\left(x_{m_{p}}, x_{n_{p}-1}, x_{n_{p}-1}, \frac{t}{2}\right) \\
& \geqslant \mathscr{M}\left(x_{m_{p}-1}, x_{n_{p}-1}, x_{m_{p}}, \frac{t}{2}\right) \diamond \boldsymbol{\varepsilon} \\
& \rightarrow \boldsymbol{\varepsilon} \quad \text { as } p \rightarrow \infty
\end{aligned}
$$

Hence

$$
\begin{aligned}
& \lim _{p} \int_{0}\left(\frac{1}{\mathscr{M}\left(x_{m_{p}-1}, x_{\left.n_{p}-1, x_{n_{p}-1}, t\right)}\right.}-1\right) \phi(s) d s<\int_{0}^{\frac{\varepsilon}{1-\varepsilon}} \phi(s) d s \quad \text { and } \\
& \lim _{p} \int_{0}^{\mathscr{N}\left(x_{m_{p}-1}, x_{n-1}, x_{n p}, t\right)} \phi(s) d s<\int_{0}^{\varepsilon} \phi(s) d s
\end{aligned}
$$

By using (3.1), (3.3) and (3.6) we have

$$
\begin{aligned}
\int_{0}^{\left(\frac{\varepsilon}{1-\varepsilon}\right)} \phi(s) d s & \leq \int_{0}^{\left(\frac{1}{\mathscr{M}\left(x_{m_{p}}, x_{n}, x_{n}, t\right)}-1\right)} \phi(s) d s \\
& \leq k \int_{0}^{\left(\frac{1}{\mathscr{M}\left(x_{m_{p}-1}, x_{n_{p}-1}, x_{n_{p}-1}, t\right)}-1\right)} \phi(s) d s \\
& <k \int_{0}^{\left(\frac{\varepsilon}{1-\varepsilon}\right)} \phi(s) d s \text { and }
\end{aligned}
$$

$$
\begin{aligned}
\int_{0}^{\mathcal{E}} \phi(s) d s & \leq \int_{0}^{\mathscr{N}\left(x_{m_{p}}, x_{n_{p}}, x_{n_{p}}, t\right)} \phi(s) d s \\
& \leq k \int_{0}^{\mathscr{N}\left(x_{m_{p}-1}, x_{n_{p}-1}, x_{n_{p}-1}, t\right)} \phi(s) d s \\
& <k \int_{0}^{\mathcal{E}} \phi(s) d s
\end{aligned}
$$

which is a contraction so $\left(x_{n}\right)$ is a Cauchy sequence. Since $X$ is complete so there exist $z \in X$ such that $\mathrm{x}_{n} \rightarrow \mathrm{z}$. Now, using (3.1) and taking $n \rightarrow \infty$ we get,

$$
\begin{array}{r}
\int_{0}^{\left(\frac{1}{\mathscr{M}\left(T z, x_{n+1}, x_{n+1}, t\right)}-1\right)} \phi(s) d s \leq k \int_{0}^{\left(\frac{1}{\mathscr{M}\left(z, x_{n}, x_{n}, t\right)}-1\right)} \phi(s) d s \\
<k \int_{0}^{\left(\frac{1}{\mathscr{M}(z, z, z, t)}-1\right)} \phi(s) d s=0
\end{array}
$$

and

$$
\begin{aligned}
\int_{0}^{\mathscr{N}\left(T z, x_{n+1}, x_{n+1}, t\right)} \phi(s) d s \leq & k \int_{0}^{\mathscr{N}\left(z, x_{n}, x_{n}, t\right)} \phi(s) d s \\
& <k \int_{0}^{\mathscr{N}(z, z, z, t)} \phi(s) d s=0
\end{aligned}
$$

which implies that $\mathscr{M}(\mathrm{Tz}, \mathrm{z}, \mathrm{z}, \mathrm{t})=1$ and hence $\mathrm{Tz}=\mathrm{z}$, that is, $z$ is fixed point of T. For uniqueness let us suppose that $y$ and $z$ are two distinct fixed points of $T$ then from(3.1)

$$
\begin{aligned}
\int_{0}^{\left(\frac{1}{\mathscr{M}(y, z, z, t)}-1\right)} \phi(s) d s & =\int_{0}^{\left(\frac{1}{\mathscr{M}(T y, T, T, T, t)}-1\right)} \phi(s) d s \\
\leq k & \int_{0}^{\left(\frac{1}{\mathscr{M}(y, z, z, t)}-1\right)} \phi(s) d s \\
\text { and } \int_{0}^{\mathscr{N}(y, z, z, t)} \phi(s) d s & =\int_{0}^{\mathscr{N}(T y, T z, T z, t)} \phi(s) d s \\
\leq & k \int_{0}^{\mathscr{N}(y, z, z, y)} \phi(s) d s
\end{aligned}
$$

since $\mathrm{k}<1$ so $\left(\frac{1}{\mathscr{M}(y, z, z, t)}-1\right)=0$ and $\mathscr{N}(y, z, z, t)=0$. Which implise $\mathscr{M}(\mathrm{y}, \mathrm{z}, \mathrm{z}, \mathrm{t})=1$ and $\mathscr{N}(\mathrm{y}, \mathrm{z}, \mathrm{z}, \mathrm{t})=0$ or $y=z$. 


\section{References}

[1] Al-Thagafi. A and Shahzad. N, "Generalized INonexpansive Self maps and Invariant Approximations", Acta Mathematica Sinica, English Series, 24(2008), 5, 867-876.

[2] Atanassov. K, "Intuitionistic fuzzy Sets" Fuzzy sets and Systems, 20 (1986), 87-96.

[3] Branciari. A, "A fixed point theorem for mappings satisfying a general contractive condition of integral type" Int. J. Math. Sci. 29(2002), 531-536.

[4] George.A and Veeramani. P, "On Some results in fuzzy metric spaces", Fuzzy sets and Systems, 64(1994), 395399.

[5] Gregori. V and Sapena. A, "On fixed-point theorems in fuzzy metric spaces", Fuzzy sets and Systems, 125(??), 2002, 245-252.

[6] Jeyaraman. M and Balamurugan. R, "Fixed point theorems in intuitionistic fuzzy metric spaces by using occasionally weakly compatible maps" Elixir Adv. Pure Math. 70(2014), 24097 - 24102.

[7] Kramosil. O and Michalek. J, "Fuzzy metric and statistical metric spaces", Ky-bernetics, 11 (1975), 330-334.

[8] Park. J. H, "Intuitionistic fuzzy metric spaces", Chaos, Solitons and Fractals, 22 (2004), 1039-1046.

[9] Singh. B and Chughan.M.S, "Generalized fuzzy metric space and Fixed point theorem",Bull. Cal. Math. Soc., 89(1997), 457-460.

[10] Singh. B and Chughan. M. S, "Common fixed points of compatible maps in fuzzy metric spaces", Fuzzy sets and systems, 115(2000), 471-475.

[11] Surjeet Singh Chauhan, "Common fixed point theorem in M-Fuzzy metric space satisfying integral type contractive conditions", Advanced Science Research, 2013, 4(2), 212-218.

[12] Zadeh, L.A., "Fuzzy sets", Information and Control, 8 (1965), 338-353.

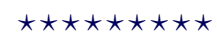

$\operatorname{ISSN}(\mathrm{P}): 2319-3786$

Malaya Journal of Matematik

$\operatorname{ISSN}(\mathrm{O}): 2321-5666$

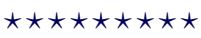

